\title{
A latex slide agglutination test for rapid detection of antimyeloperoxidase antibody
}

\author{
K H Ko, S S Lee, J W M Lawton
}

\begin{abstract}
Aim-To develop and test a new latex slide agglutination test (MPO-LSAT) to detect antimyeloperoxidase (anti-MPO) antibody in serum.

Methods-Latex bead coating was adjusted to give maximum sensitivity by attending to latex size, MPO to latex ratio for coupling, ratio of diluted serum to MPO-latex, reaction time and temperature for coupling, and reaction time for agglutination. Inhibition studies were performed using MPO, proteinase 3, bactericidal/permeability increasing protein, and lactoferrin.

Results-There was very good correlation between this test and the conventional anti-MPO enzyme linked immunosorbent assay (ELISA): $81 \%$ of sera positive in the ELISA were positive by MPO-LSAT. MPO-LSAT results correlated better with IgM anti-MPO than with IgG anti-MPO. Conclusions-MPO-LSAT is a simple diagnostic test that is potentially useful in the clinical laboratory as a rapid screening tool for vasculitic diseases. (F Clin Pathol 1999;52:770-772)
\end{abstract}

Keywords: latex agglutination; antineutrophil cytoplasmic antibody; anti-MPO antibody

Antineutrophil cytoplasmic antibody (ANCA) comprises a group of autoantibodies first reported by Davies et al in $1982 .{ }^{1}$ Its association with systemic vasculitis and the classification into C-ANCA and P-ANCA forms have become established. ${ }^{2} \mathrm{Up}$ to now, indirect immunofluorescence has remained the standard method for ANCA detection, using cytocentrifuged, alcohol fixed human neutrophils as substrate. Myeloperoxidase (MPO), one major target antigen of ANCA found in some vasculitic diseases, such as necrotising and crescentic glomerulonephritis and microscopic polyarteritis, ${ }^{34}$ is detected by enzyme linked immunosorbent assay (ELISA). Some patients with anti-MPO, in particular those with pulmonary-renal syndrome, have life threatening disease. Early detection of these antibodies is extremely useful in establishing an early diagnosis and guiding prompt treatment.

Passive latex agglutination is a simple method widely used in diagnostic laboratories. In this study we set out to develop a new latex slide test to detect anti-MPO, and to compare its performance with the established ELISA system.

\section{Methods}

SERUM SAMPLES AND IMMUNOFLUORESCENCE Sera received at the immunology laboratory, Queen Mary Hospital, Hong Kong were first screened for ANCA by immunofluorescence. Positive results were distinguished by their pattern: either perinuclear staining (P-ANCA) or cytoplasmic staining (C-ANCA). Seventy three P-ANCA positive sera (43 anti-MPO IgG positive, 30 anti-MPO IgG negative), 40 C-ANCA positive sera, and 40 ANCA negative sera from normal individuals were collected for testing. The method of immunofluorescence was as described in the First International Workshop on ANCA. ${ }^{5}$

\section{ANTI-MPO ELISA}

Neutrophil MPO (Calbiochem) was coated onto Immulon 2 microtitre plates (Dynex) at a concentration of $1.25 \mu \mathrm{g} / \mathrm{ml}$ in $0.05 \mathrm{M}$ bicarbonate buffer, $\mathrm{pH}$ 9.5. After washing three times with $0.05 \%$ Tween 20 in phosphate buffered saline (PBS) and blocking with $1 \%$ bovine serum albumin in PBS with $0.05 \%$ Tween 20, $100 \mu \mathrm{l}$ serum diluted $1: 40$ in the same buffer were added and incubated for one hour at $25^{\circ} \mathrm{C}$, followed by washing. Alkaline phosphatase conjugated goat antihuman IgG or IgM (Biosource) diluted 1:1000 was added and incubated for one hour at $25^{\circ} \mathrm{C}$, followed by the substrate p-nitrophenyl phosphate for 15 minutes. The absorbance was read at 405 $\mathrm{nm}$ optical density. Results were expressed as a percentage of an international standard reference serum, which had been serially diluted to obtain a standard curve in each assay. Normal cut off value was established by calculating the mean per cent plus 3 SD of 56 normal samples.

\section{ANTI-MPO LATEX TEST}

The latex bead coating method was modified from that of Goode et al. ${ }^{6}$ The following conditions were adjusted to give maximum sensitivity: latex size; MPO to latex ratio for coupling; ratio of diluted serum to MPO-latex; reaction time and temperature for coupling; reaction time for agglutination. Inhibition studies were performed using MPO, proteinase 3, bactericidal/permeability increasing protein, and lactoferrin.

One hundred microlitres of MPO at 0.5 $\mathrm{mg} / \mathrm{ml}$ concentration (Calbiochem) were mixed with $50 \mu \mathrm{l} 10 \%$ (vol/vol) carboxylate modified latex beads, $0.8 \mu \mathrm{m}$ diameter (Sigma) in $0.1 \mathrm{M}$ phosphate buffer for 10 minutes at $25^{\circ} \mathrm{C}$. Then $50 \mu \mathrm{l}$ of $15 \%$ (wt/vol) 1 -cyclohexyl3-(2-morpholinoethyl)carbodiimide metho-ptoluenesulphonate (Sigma) were added to covalently bind MPO to the latex particles. The mixture was put on a roller-mixer for two hours
Accepted for publication 1 June 1999 

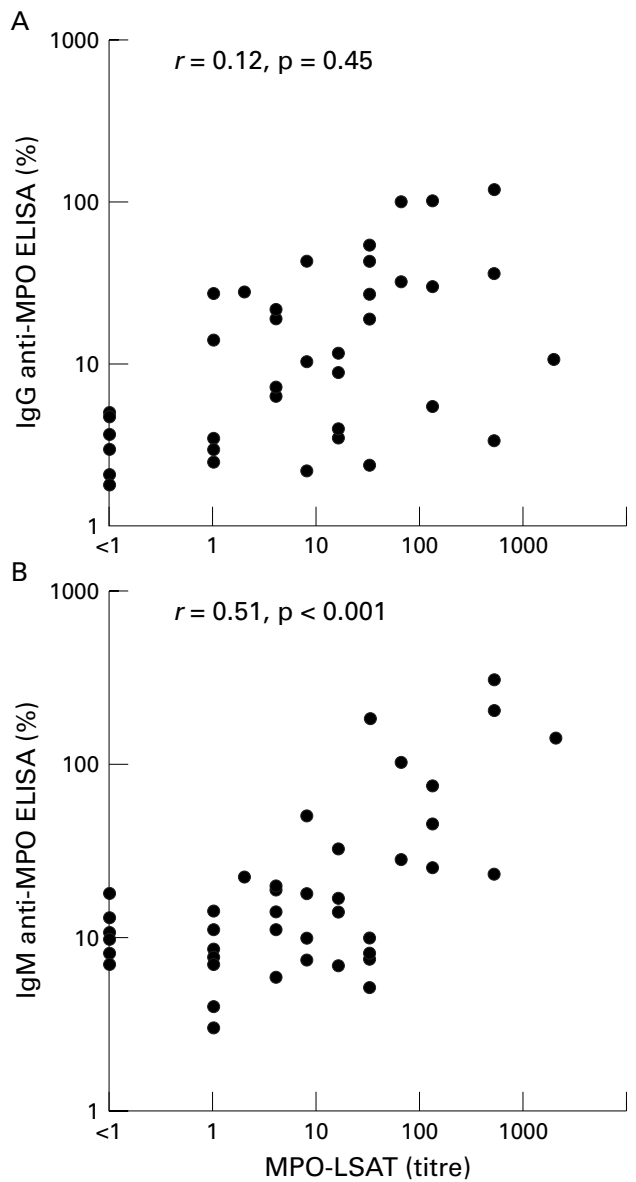

Figure 1 Correlation between myeloperoxidase (MPO) latex slide agglutination test (MPO-LSAT) titre and IgG/IgM enzyme linked immunosorbent assay (ELISA) (binding activity expressed as a percentage of the standard reference serum) in serum samples positive for IgG anti-MPO. Correlation coefficient $(r)$ and $p$ values were calculated from the Pearson correlation test.

at $25^{\circ} \mathrm{C}$. After washing three times in glycine buffer $(1 \%$ bovine serum albumin in glycine buffered saline, $\mathrm{pH} 8$, with $0.1 \%$ Tween 20 ), coated latex was suspended in glycine buffer at a concentration of $0.5 \%$. MPO coated latex was stored at $4^{\circ} \mathrm{C}$ until use.

Serum anti-MPO was detected by passive slide agglutination. Briefly, $5 \mu \mathrm{l}$ of MPO-latex were added to $10 \mu \mathrm{l}$ diluted serum on the dark background glass slide. After rotating the glass slide for two minutes by hand, the agglutination was read by eye. The strength of agglutination was graded as,$-+/-,+,++$, or +++ . The titre of serum was defined as the dilution which gave a + result.

All sera were tested neat and at $1 / 4$ dilution to exclude "prozone effect." Positive sera were then further titrated by twofold serial dilution to determine the titre.

\section{Results}

The development of the new anti-MPO latex agglutination test required optimisation of the assay conditions. The single most important factor was establishment of the optimum MPO to latex concentration ratio. Owing to potential batch variation, titration of MPO was necessary for each new batch. For this batch of
Table 1 Summary of results of enzyme linked immunosorbent assay (ELISA) versus myeloperoxidase latex slide agglutination test (MPO-LSAT) for the detection of antimyeloperoxidase antibodies in 153 sera

\begin{tabular}{lll}
\hline & $\begin{array}{l}\text { IgG ELISA } \\
\text { positive }\end{array}$ & $\begin{array}{l}\text { IgG ELISA } \\
\text { negative }\end{array}$ \\
\hline $\begin{array}{l}\text { MPO-LSAT positive } \\
\text { MPO-LSAT negative }\end{array}$ & $\begin{array}{l}\text { 85 } \\
\text { IgM ELISA }\end{array}$ & $\begin{array}{l}\text { IgM ELISA } \\
\text { negative }\end{array}$ \\
\hline & 18 & 17 \\
\hline $\begin{array}{l}\text { MPO-LSAT positive } \\
\text { MPO-LSAT negative }\end{array}$ & 0 & 118
\end{tabular}

MPO, a combination of $100 \mu \mathrm{l}$ MPO (0.5 $\mathrm{mg} / \mathrm{ml}$ ) and $50 \mu \mathrm{l}$ latex $(10 \%)$ gave the best results. The stability of $\mathrm{MPO}$-latex during storage was monitored by sequential testing of aliquots of standard anti-MPO positive serum, stored at $-70^{\circ} \mathrm{C}$. MPO-latex was found to be stable at $4^{\circ} \mathrm{C}$, without any change of titre of reference serum, for at least six months (data not shown).

In terms of positivity and negativity, correlation of the MPO-LSAT with the conventional ELISA was excellent. However, absolute values correlated only weakly in the IgM assay (fig 1). MPO-LSAT did not react with normal human sera, anti-MPO negative P-ANCA sera, and C-ANCA positive sera except for one sample (table 1). The latter belonged to a C-ANCA positive patient and tested strongly positive for anti-MPO IgM (binding activity $>100 \%$ ). In P-ANCA positive sera, 35 of 43 anti-MPO IgG positive samples were detected by MPO-LSAT. Agglutination negative samples had low MPO binding in the IgG ELISA (binding activity $<6 \%$ ) and no significant activity in the $\operatorname{IgM}$ anti-MPO ELISA. Thus, using MPO IgG ELISA results as the reference, the sensitivity of MPO-LSAT was $81 \%$.

It was confirmed that MPO-latex was specifically agglutinated by anti-MPO, but not by anti-PR3, anti-bactericidal/permeability increasing protein (BPI) or anti-lactoferrin. This agglutination ability of anti-MPO could be inhibited by MPO but not by PR3, BPI, or lactoferrin.

A sample strongly positive in MPO-LSAT was further evaluated by serial dilution. MPOlatex did not agglutinate in the neat serum, owing to the prozone effect. In view of this phenomenon, a $1 / 4$ dilution of test serum was routinely included in MPO-LSAT.

Finally, MPO-latex was agglutinated better by IgM anti-MPO than by IgG. MPO-LSAT titre $(r=0.51, \mathrm{p}<0.001)$ correlated with $\operatorname{IgM}$ anti-MPO binding in ELISA but less so with IgG anti-MPO ( $r=0.12, \mathrm{p}=0.45)($ fig 1$)$.

\section{Discussion}

Patients with anti-MPO associated vasculitis often present with severe illness, such as acute renal failure. Access to early and reliable testing is crucial in guiding subsequent clinical management. This is particularly important in Hong Kong where anti-MPO is the commonest autoantibody found in patients with small vessel vasculitis. The development of the latex 
test therefore contributes towards a more efficient and effective system in the diagnosis of anti-MPO positive vasculitis.

The new MPO-LSAT is a simple procedure, the performance of which is comparable with the conventional assay by ELISA. It is a rapid diagnostic tool, the results of which can be generated within minutes. Technically, the test is simple to perform and results are easy to read. The procedure's speed and simplicity are particularly important when an urgent result is needed in a clinical laboratory.

To exclude confounding results in the presence of other autoantibodies, we tested 20 antinuclear antibody positive sera and 20 rheumatoid factor positive sera. All were negative by IgG anti-MPO ELISA. Two rheumatoid factor positive sera proved to be positive in the MPOLSAT and the IgM anti-MPO ELISA. Thus rheumatoid factor may occasionally give rise to false positive results.

MPO-LSAT has a very high specificity and correlates positively with anti-MPO ELISA, especially with the ELISA for IgM. IgM anti-MPO antibody had been reported to be associated with clinical manifestation such as pulmonary haemorrhage $\mathrm{e}^{7}$ but it is not commonly assayed in clinical laboratories. MPOLSAT may prove to be a useful laboratory diagnostic tool in $\operatorname{IgM}$ anti-MPO associated disease. The stability of the test reagent and reproducibility of results add value to the simple assay system. Its preferential reaction with IgM anti-MPO would need to be further investigated to determine its clinical relevance.

1 Davies DJ, Moran JE, Niall JF, et al. Segmental necrotising glomerulonephritis with antineutrophil antibody: possible arbovirus aetiology? $B M \mathcal{F}$ 1982;285:606.

2 Kallenberg CG, Brouwer E, Weening JJ, et al. Antineutrophil cytoplasmic antibodies: current diagnostic and pathophysiological potential. Kidney Int 1994;46:1-15.

3 Lee SS, Adu D, Thompson RA. Anti-myeloperoxidase antibodies in systemic vasculitis. Clin Exp Immunol 1990;79. 41-6.

4 Cohen Tervaert JW, Goldschmeding R, Elema JD, et al. Association of autoantibodies to myeloperoxidase with different forms of vasculitis. Arthritis Rheum 1990;33:126472 .

5 Wiik A. Delineation of a standard procedure for indirect immunofluorescence detection of ANCA. Acta Pathol Mimmunofluorescence detection of ANCA. Acta

6 Goode NP, Davison AM, Gowland G, et al. Preparation, purification and analysis of BSA-coated latex particles for in vivo studies. F Immunol Methods 1986;92:31-5.

7 Esnault VL, Ronda N, Jayne DR, et al. Association of ANCA isotype and affinity with disease expression. $\mathcal{F}$ Autoimmun 1993;6:197-205. 